\title{
Research on Ladder Wave - specific Harmonic Elimination Technology for Cascade $\mathrm{H}$ - bridge Converter
}

\author{
Suting Gao \\ School of North China Electric Power University, Baoding 071000, China \\ 377616544@qq.com
}

\begin{abstract}
In this paper, the low-order harmonic elimination problem of H-bridge unit number 3 is explored with the cascade H-bridge converter. The initial value is calculated by using the method of vertical bisector reference sinusoidal signal, and then using the principle of least squares The Gauss-Newton method is iteratively solved, and the three switching angles are respectively, and the corresponding THD is $11.39 \%$. Through the dichotomy to discuss the angle of the modulation ratio when the switching angle is resolved, the corresponding THD range is.
\end{abstract}

Key words: least square method, Gauss - Newton method, dichotomy

\section{Introduction}

The specific harmonic elimination pulse width modulation technique by selecting a specific switching moment, while satisfying the desired output fundamental voltage, eliminates the selected lower harmonics, thereby improving the output voltage waveform quality. In this paper, for the elimination of low harmonics, by controlling the size of the switching angle so that the specific harmonic amplitude of 0 , and then solve the solution to test to observe whether the specific harmonic is completely eliminated. In determining the range of the sweep angle, consider using dichotomy, but in the process of using dichotomy to narrow the range of exhaustive.

\section{Model}

\section{1 control switch angle specific low-order harmonic model}

\subsubsection{Establishment of Nonlinear Equations}

The converter module unit often uses a 3-level output for the H-bridge converter unit. Since the cascade multi-level converter output voltage vac is the superposition of the output voltage vaci of each $\mathrm{H}$-bridge converter unit, the increase in the number of levels allows The output of the stepped voltage is closer to the sine wave. 


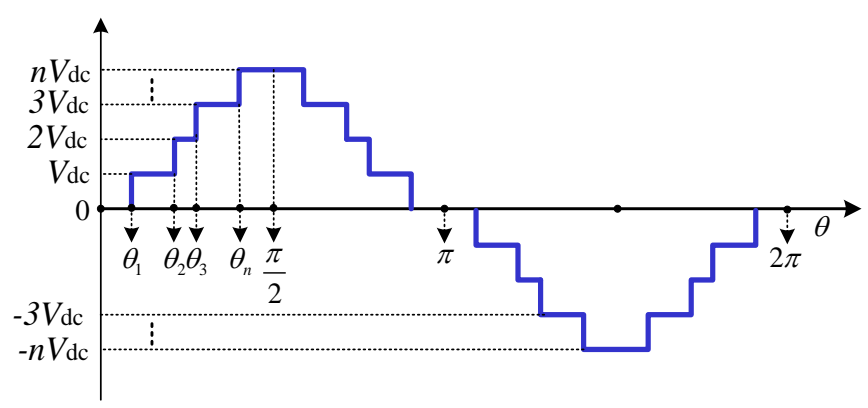

Figure 1: Cascaded multi-level converter output voltage

For the n-type converter system of n H-bridge converter units, the number of specific harmonics can be eliminated (n-1) if the desired fundamental voltage amplitude is satisfied, then the amplitude of these harmonics is 0 , Through this principle can be obtained by eliminating the harmonic formula is:

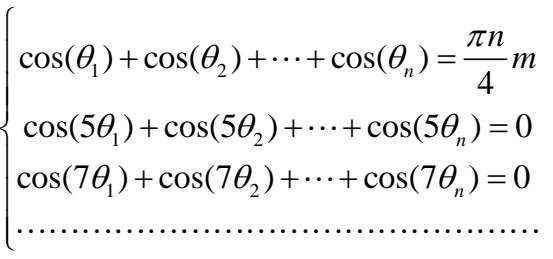

\subsubsection{Solving model of nonlinear equations}

The key to solving the nonlinear equations includes the selection of high quality initial values and the efficient iterative algorithm.

\section{(1) selection of initial values}

In this paper, the output voltage of the first quarter of the reference signal is divided into equal parts. In the first aliquot, the area is equal to the area of the area of the reference sinusoidal signal and the time axis, And the center of the rectangular wave is the center of the partition, the rising value of the rising edge of the PWM wave in the 1/4 cycle can be the initial value of the SHEPWM nonlinear system. It was verified that the convergence rate was the fastest and the number of iterations was the least.

Push the formula as follows:

$$
\theta_{k}^{0}=\arcsin \left(\frac{k}{n+0.5}\right) \quad(k=1,2,3, \cdots, n)
$$

Table 1: $n=3, m=0.8$ when the initial value of the switch angle

\begin{tabular}{ccc}
\hline$\theta_{1}^{0}$ & $\theta_{2}{ }^{0}$ & $\theta_{3}{ }^{0}$ \\
\hline $16.6015^{\circ}$ & $34.8499^{\circ}$ & $58.9973^{\circ}$ \\
\hline
\end{tabular}

\section{(2) Gauss - Newton iterative solution based on least squares method}

Principle 1: Nonlinear least squares estimation:

The general form of a nonlinear system of expressions with $n$ unknowns of $n$ equations is:

$$
Z=f(X)+\Delta
$$

The error equation for the nonlinear model corresponding to an estimate of the parameter is:

$$
E(\hat{X})=f(\hat{X})-Z
$$


The sum of squares of residuals is:

$$
E^{T} E=\|E(\hat{X})\|^{2}=\|f(\hat{X})-Z\|^{2}=(f(\hat{X})-Z)^{T}(f(\hat{X})-Z)
$$

If the estimate makes the square of the residuals reach the minimum (the distance between the observation space and the solution space is the smallest), then a least squares estimate is called the objective function:

$$
T(\hat{X})=E^{T} E=\min
$$

Principle 2: Solve by Gauss-Newton method

The estimation of the nonlinear least squares method is to solve the unknown parameters according to certain criteria. Since the Gauss-Newton iterative method [6] has the characteristics of fast convergence and high accuracy for the nonlinear model, the Gauss-Newton iterative method [3] is used to solve the parameters. The basic idea is to replace the nonlinear model with a linear model with a Taylor series expansion approximation after a given initial value, and then to the model convergence by repeated iterations, even if the residual sum of the original model is minimized. [4] Then according to the least squares principle, we can see:

$$
X^{1}=X^{0}+\left(B^{T}\left(X^{0}\right) B\left(X^{0}\right)\right)^{-1} B^{T}\left(X^{0}\right)\left(Z-f\left(X^{0}\right)\right)
$$

Where B is the correction factor, and then the next iteration is assumed to be the approximation, and the iteration formula is obtained:

$$
X^{i+1}=X^{i}+\left(B^{T}\left(X^{i}\right) B\left(X^{i}\right)\right)^{-1} B^{T}\left(X^{i}\right)\left(Z-f\left(X^{i}\right)\right)
$$

The value of the objective function is equal when two iterations are adjacent, ie the iteration ends.

\section{(3) specific examples to solve}

When $\mathrm{n}=3, \mathrm{~m}=0.8$, the Matlab model can be used to solve the model can be eliminated 5,7 harmonic switching angle:

Table 2: $n=3, m=0.8$ to eliminate the 5, 7 harmonic corresponding to the switch angle

\begin{tabular}{ccc}
\hline$\theta_{1}$ & $\theta_{2}$ & $\theta_{3}$ \\
\hline $29.2355^{\circ}$ & $54.4383^{\circ}$ & $64.4844^{\circ}$ \\
\hline
\end{tabular}

\section{2 the output voltage corresponding to the THD solution}

\subsubsection{The establishment of the model}

According to the above model can be solved to eliminate the specific waveform of the switching angle of the value, and then into the formula (1) to get the odd number of odd harmonics $V_{5}, V_{7}, V_{11}, \cdots \cdots, V_{43}, \cdots \cdots$

And then based on the ladder wave specific harmonic elimination technology output voltage waveform quality, the following formula can be obtained by the total harmonic distortion rate THD:

$$
T H D=\sqrt{\frac{\sum_{i=6 k+1,2, \cdots}^{\infty} V_{i}^{2}}{V_{1}^{2}}} \times 100 \%=\sqrt{\frac{V_{5}^{2}+V_{7}^{2}+V_{11}^{2}+\cdots+V_{43}^{2}+\cdots}{V_{1}^{2}}} \times 100 \%
$$




\subsubsection{Specific examples of solving}

When $\mathrm{n}=3, \mathrm{~m}=0.8$ :

Table 3: Amplitude of each odd harmonic output voltage

\begin{tabular}{cccccc}
\hline odd harmonic & $V_{1}$ & $V_{3}$ & $V_{5}$ & $V_{7}$ & $V_{9}$ \\
\hline Output Voltage & 2.4000 & 0.8023 & 0.0000 & 0.0000 & 0.2156 \\
\hline$V_{11}$ & $V_{13}$ & $V_{15}$ & $V_{17}$ & $V_{19}$ & $V_{21}$ \\
\hline 0.1445 & 0.1413 & 0.0256 & 0.0505 & 0.0728 & 0.0150 \\
\hline
\end{tabular}

As can be seen from the results: 5,7 harmonic has been completely eliminated, the largest fundamental amplitude. Although the other high-order harmonics, although some shocks, but the overall trend of decreasing.

And then according to Table 3 to eliminate the 5,7 harmonic output voltage, into the formula (3) to obtain the corresponding total voltage harmonic distortion THD:

$$
\begin{aligned}
T H D & =\sqrt{\frac{0.2156^{2}+0.1445^{2}+0.1413^{2}+0.0256^{2}+\cdots}{2.4000^{2}}} \times 100 \% \\
& =11.39 \%
\end{aligned}
$$

\section{Conclusions}

Model Advantages:

1. The improved Gaussian-Newton iteration reduces the dependence on the initial value.

2. The improved Gauss-Newton method reduces the number of iterations.

3. Weighted optimization control The power equalization model improves the shortcomings of the traditional method of long equalization period and high distortion rate.

Model Disadvantages:

1. The Gauss-Newton method and the improved Gauss-Newton must have a solution under the full rank of the matrix, otherwise it is morbid.

\section{References}

[1] Chen Fei.Study on several classes of algorithms for large-scale nonlinear equations [D]. China University of Mining and Technology, 2014.

[2] FEI Wan-min, RUAN Xin-bo, ZHANG Yan-li, ZHU Rong.Study on the initial value of multi-level inverter-specific harmonic elimination pulse width modulation method [J]. Proceeding of the CSEE, 2007,13: 87-92.

[3] Qin Xiaojun. Accelerated iterative method for nonlinear equations [J]. Shanghai Jiaotong University, 2010.

[4] FEI Wan-min, ZHANG Yan-li, RUAN Xin-bo, YAO Wen-xi, LU

Zheng-yu.Preparation of SHEPWM Nonlinear Equations for Three-level Inverter [J].

Proceeding of the CSEE, 2008,06: 62-68.

[5] ZHU Si-guo, OUYANG Hong-lin, ZHU Ying-hao. Iterative Harmonic Elimination Method for H-Bridge Cascade Inverter [J]. High Voltage Technology, 2011,02:

318-324

[6] Su Yi. Semi-periodic symmetrical SHEPWM technology switching angle method [D]. Wuhan University, 2012. 